\title{
Câncer infantil: perfil epidemiológico em população atendida por hospital de referência no Piauí
}

\author{
Childhood cancer: epidemiological profile of a population met by a reference hospital in \\ Piauí
}

\begin{abstract}
Cáncer infantil: perfil epidemiológico en una población atendida por un hospital de referencia en Piauí
\end{abstract}

\begin{abstract}
Mayra Aparecida Santos Araújo ${ }^{1 *}$, Gabriel Lima Jurema ${ }^{1}$, Allan Dellon da Silva ${ }^{1}$, Edinaldo Gonçalves de Miranda ${ }^{1}$, Maria Aline Ferreira de Cerqueira ${ }^{1}$, Lucielma Salmito Soares Pinto ${ }^{1}$, Luciana Tolstenko Nogueira'.
\end{abstract}

\section{RESUMO}

Objetivo: Avaliar o perfil epidemiológico do câncer infantil em população atendida por hospital de referência no estado do Piauí durante o período de janeiro/2017 a dezembro/2018. Métodos: Estudo descritivo e transversal com avaliação da base de dados de um hospital de referência em oncologia pediátrica no Piauí. Foram incluídos 196 pacientes entre 0 a 19 anos que receberam tratamento oncológico entre janeiro/2017 e dezembro/2018. As variáveis analisadas foram sexo, idade, tipo de neoplasia e procedência dos pacientes. Resultados: Dos 196 pacientes avaliados, 52\% eram do sexo masculino. Segundo a faixa etária, $45 \%$ tinham idade superior a 10 anos. A maioria teve diagnóstico de leucemia (43.4\%), seguida de tumor renal (16.4\%) e ósseo (10.2\%). Quanto à procedência, $85 \%$ dos pacientes eram do Piauí e 15\% do Maranhão. Conclusão: Entre a população avaliada, observou-se que o câncer infantil teve maior incidência entre indivíduos do sexo masculino, procedentes do interior do Piauí, com idade acima de 10 anos sendo a leucemia o tipo de neoplasia mais frequente.

Palavras-chave: Câncer infantil, Cancerologia, Epidemiologia.

\section{ABSTRACT}

Objectives: To evaluate the epidemiological profile of childhood cancer in the population met by a reference hospital in the state of Piauí during the period from January/2017 to December/2018. Methods: Crosssectional and descriptive study with assessment of the database from a hospital reference in pediatric oncology in Piauí. The sample included 196 patients aged between 0 and 19 years who received oncological treatment between January 2017 and December/2018. The variables analyzed were: gender, age, type of neoplasm and origin of patients. Results: Of the 196 patients evaluated, $52 \%$ were male. According to age range, $45 \%$ were aged over 10 years. The majority had diagnosis of leukemia $(43.4 \%)$, followed by renal $(16.4 \%)$ and bone (10.2\%) tumor. Regarding origin, $85 \%$ of the patients were from Piauí and $15 \%$, from Maranhão. Conclusion: Among the population evaluated, the childhood cancer had a higher incidence among male individuals, coming from the countryside of Piauí, aged over 10 years and with leukemia as the most common type of neoplasm.

Keywords: Childhood cancer, Medical oncology, Epidemiology.

\section{RESUMEN}

Objetivos: Evaluar el perfil epidemiológico del cáncer infantil en la población atendida por el hospital de referencia en el estado de Piauí, durante el período de enero/2017 a diciembre/2018. Métodos: Estudio descriptivo y transversal con la evaluación de la base de datos de un hospital de referencia en oncología pediátrica en Piauí. Se incluyeron 196 pacientes de edades comprendidas entre 0 y 19 años que recibieron tratamiento oncológico, entre enero/2017 y diciembre/2018. Se analizaron las variables: sexo, edad, tipo de neoplasia y la procedencia de los pacientes. Resultados: De los 196 pacientes evaluados, el $52 \%$ era del sexo masculino. Según rango de edad, el 45\% tenían más de 10 años. La mayoría tenía el diagnóstico de

1Universidade Estadual do Piauí (UESPI), Teresina - PI. *E-mail: mayra_bkaraujo@hotmail.com 
leucemia (43,4\%), seguido por tumor renal (16,4\%) y del hueso (10,2\%). En relación con el origen, el $85 \%$ de los pacientes eran del Piauí y 15\% del Maranhão. Conclusión: En la población evaluada, se observó que el cáncer infantil tuvo una mayor incidencia en el sexo masculino, procedente del interior del Piauí, con edad superior a 10 años y la leucemia, el tipo más común de neoplasia.

Palabras clave: Cáncer infantil, Oncología médica, Epidemiología

\section{INTRODUÇÃO}

Ao se falar de câncer infantil, estamos nos referindo a todas as neoplasias malignas que acometem crianças e adolescentes de 0 a 19 anos (BRASIL, 2016). Trata-se de um grupo de doenças onde ocorre proliferação descontrolada de células anormais e pode surgir em qualquer parte do organismo (LIMA IM, 2018).

Os tipos mais comuns na infância e adolescência são as leucemias, linfomas, tumores do sistema nervoso central (SNC), neuroblastomas, rabdomiossarcomas, tumor de Wilms, retinoblastomas e tumores ósseos (SILVA MGP, et al., 2018). O câncer infanto-juvenil tem sua origem, predominantemente, em células embrionárias, com período de latência curto e rápido crescimento (BRASIL, 2017).

E trata-se de uma doença que pode apresentar ampla diversidade de sinais e sintomas, dentre eles estão: aparecimento de massa abdominal, linfonodomegalia, alterações no hemograma, déficit neurológico específico, reflexo pupilar branco, dor nos membros, entre outros (SILVA MGP, et al., 2018).

Ao se realizar o diagnóstico precoce a chance de cura pode ser de até 70\% (FERNANDES LMS e SOUZA AM, 2019). No entanto, o diagnóstico precoce é difícil de se realizar na prática, pois os sinais e sintomas podem ser confundidos com doenças e situações comuns na infância, porém é algo que deve ser buscado já que a prevenção ainda é um desafio nesse contexto (DELFIN BS, et al., 2018).

Nos adultos podemos definir alguns fatores de risco ambientais sabidamente relacionados com o desenvolvimento de cânceres, como exposição à carcinógenos e hábitos de vida inadequados, já na criança, especialmente nos primeiros dois anos de vida, o desenvolvimento de neoplasias tem forte relação com fatores genéticos herdados dos pais ou mutações adquiridas de causa não estabelecida.

Há algumas associações que têm sido observadas com frequência, como maior ocorrência de leucemia em portadores de síndrome de Down, de tumores do SNC e de sarcomas em crianças com neurofibromatose e de tumor renal em pacientes com malformações do trato genitourinário, por exemplo (BRASIL, 2017).

O câncer é uma das principais causas de mortalidade em diversos países, causando impacto na saúde pública (SILVA MGP, et al., 2018). Na Colômbia, por exemplo, o câncer representa a terceira principal causa de morte em crianças de 1 a 14 anos de idade (WURTTEMBERGER OR, 2016). Em nosso país essa doença foi responsável por 2.800 óbitos no ano de 2013 (FERNANDES LMS e SOUZA AM, 2019).

As neoplasias do SNC apresentam a maior taxa de mortalidade $(36,27 \%)$, em seguida estão neuroblastoma (32,13\%), leucemias $(29,31 \%)$ e neoplasias dos tecidos moles $(21,56 \%)$ (SPIRONELLO RA, et al., 2020). A neoplasia mais comumente diagnosticada entre crianças e adolescentes é a leucemia do tipo linfoblástica aguda, sendo o sexo masculino o mais afetado pela doença (VIEIRA AF, et al., 2017).

Ademais, no Brasil, apesar do câncer infanto-juvenil representar cerca de $3 \%$ de todos os casos de câncer no país, esse foi o principal responsável de óbitos por doença entre crianças e adolescentes de 1-19 anos em 2013 (BRASIL, 2016). Um estudo realizado em Petrolina (PE) demonstrou que a faixa etária mais acometida pelo câncer infantil foi a superior a 10 anos de idade, sendo a leucemia o tipo mais frequente entre as crianças de 1 a 9 anos (SILVA MGP, et al., 2018). O tratamento de um câncer envolve procedimentos invasivos e dolorosos que geram diversos efeitos colaterais físicos e psicológicos (SANT'ANNA JL e MENDES DMLF, 2019).

No que diz respeito ao câncer infantil, o tratamento tem avançado nas últimas décadas devido às técnicas de diagnóstico precoce e evolução dos métodos terapêuticos, porém esse tratamento ainda carrega estigmas devido aos efeitos colaterais (BENEDETTI GMS, et al., 2014). Algumas possibilidades de tratamento são 
quimioterapia, radioterapia, cirurgia e transplante de células-tronco hematopoiéticas (MUTTI CF, et al., 2018). E nessa população infanto-juvenil o câncer apresenta melhor resposta à quimioterapia quando comparado com os cânceres em adultos (OLIVEIRA AT, et al., 2019).

As taxas de sobrevida dessas crianças com câncer melhoraram significativamente nas últimas décadas, principalmente nos países desenvolvidos. Por outro lado, nos países em desenvolvimento o abandono ao tratamento é uma das principais causas de insucesso da terapêutica, podendo ainda aumentar a necessidade de reabordagens cirúrgicas e tratamentos mais intensos acarretando em mais sofrimento para a criança e seus familiares, bem como o desperdício de recursos da saúde (LIMA FFS, et al., 2018).

Além disso, estudos apontam que pacientes da região norte e de algumas localidades da região nordeste, como Maranhão, sul do Piauí e oeste da Bahia, têm menos acesso ao tratamento quando comparados com pacientes da região sul e sudeste, o que demonstra desigualdade de acesso aos serviços de saúde (HANNA LMO, et al. 2016).

Ao contrário do câncer em adultos, o controle do câncer infantil não pode se basear na prevenção ou detecção pré-clínica (WURTTEMBERGER OR, 2016). Embora existam alguns estudos apontando para a correlação entre desenvolvimento do câncer infantil e exposição intrauterina a fatores de risco, não há evidência cientifica comprovada para tal afirmativa, sendo, portanto o diagnóstico precoce e tratamento oportuno e de qualidade o que vai de fato influenciar em maiores taxas de cura (BRASIL, 2017).

Com relação ao prognóstico das crianças que completam o tratamento, aproximadamente dois terços desenvolverão algum tipo de efeito tardio, e destas um terço apresentará efeito de maior complexidade ou até mesmo fatal, já que o tratamento instituído durante a fase de crescimento promove no organismo alterações que poderão se manifestar somente após o término da terapia.

Dentre esses efeitos tardios podemos citar a alteração na autoimagem decorrente da presença de cicatrizes, alopecia e baixa estatura (WHITAKER MCO, et al., 2013). A expectativa de vida dessas crianças e adolescentes está diretamente relacionada com o tempo entre o início dos sintomas e o diagnóstico.

Logo, quando o início do tratamento é feito tardiamente o prognóstico desses pacientes se torna mais reservado (MARCHI JA, et al., 2013). Diante disso, é sabido que o controle do câncer pelas autoridades de saúde e a tomada de decisões nessa área dependem de dados confiáveis.

Essa informação é crucial para conhecer a extensão real do problema, planejar estratégias de prevenção, triagem, tratamento e reabilitação, além de colaborar com uma avaliação para destinar os recursos necessários ao investimento e avaliar sistematicamente o impacto de todas as intervenções (WURTTEMBERGER OR, 2016). Pensando nisso, esse estudo teve como objetivo avaliar o perfil epidemiológico do câncer infantil em população atendida por hospital de referência no estado do Piauí.

\section{MÉTODOS}

Este é um estudo descritivo, transversal e quantitativo realizado em um hospital de referência no tratamento oncológico em pediatria no estado do Piauí, localizado na cidade de Teresina. Este hospital é referência para todo o Estado do Piauí e áreas circunvizinhas e presta assistência hospitalar e ambulatorial, com nível de atenção de média a alta complexidade. A pesquisa foi realizada com pacientes na faixa etária de 0 a 19 anos que receberam tratamento oncológico entre janeiro de 2017 e dezembro de 2018.

Para acesso às informações necessárias para a realização do estudo realizou-se a coleta de dados no período de janeiro de 2019, por meio de consulta em prontuário eletrônico, onde foram analisados sexo, idade, CID-10 da neoplasia e procedência do paciente.

Dessa maneira, foi possível analisar o perfil epidemiológico do câncer infantil no Piauí. Para análise estatística dos dados coletados, esses foram organizados e tabulados em uma planilha no programa Microsoft Excel 2010, posteriormente foi realizada a análise quantitativa dos dados de forma descritiva com as variáveis apresentadas por meio de média e as categóricas por frequências e porcentagens.

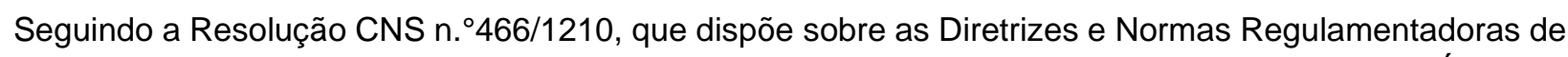
Pesquisas Envolvendo Seres Humanos (BRASIL, 2012), este estudo foi aprovado pelo Comitê de Ética em 
Pesquisa do hospital estudado e pelo Comitê de Ética em Pesquisa da instituição a qual os autores estão vinculados. E a coleta de dados iniciou somente após a sua aprovação em ambos (CAAE: 87603018.7.0000.5209 e Pareceres: 2.624.014 e 3.030.299).

\section{RESULTADOS}

No estado do Piauí foram diagnosticadas 196 crianças com câncer no período de janeiro de 2017 a dezembro de 2018. Os pacientes com este diagnóstico possuíam de 1 a 19 anos, sendo $52 \%$ do sexo masculino. Na população avaliada, 46\% estavam na faixa etária superior a 10 anos, $30 \%$ tinha entre 0 a 5 anos e $25 \%$ de 6 a 10 anos. Dentre eles, $85 \%$ eram procedentes do Piauí e 15\% do Maranhão (Tabela 1).

Tabela 1 - Dados demográficos de crianças e adolescentes com diagnóstico de câncer.

\begin{tabular}{ccc}
\hline Dado demográfico & Quantidade (n) & Frequência (\%) \\
\hline Sexo & & 52 \\
\hline Masculino & 102 & 48 \\
\hline Feminino & 94 & \\
\hline Faixa etária & & 30 \\
$0-5$ anos & 59 & 25 \\
$6-10$ anos & 48 & 45 \\
Acima de 10 anos & 89 & \\
\hline Procedência & & 85 \\
Piauí & 167 & 39,5 \\
Teresina & 66 & 60,5 \\
Interior do Piauí & 101 & 15 \\
Maranhão & 29 &
\end{tabular}

Fonte: Araújo MAS, et al., 2020.

Os tipos de câncer mais diagnosticados, independente do sexo e faixa etária, foram as leucemias com $43,4 \%$ dos casos, seguida de tumor renal $(16,4 \%)$, tumor ósseo $(10,2 \%)$, tumor do sistema nervoso central $(8,2)$ e linfomas $(7,1)$, respectivamente. Outros tipos diagnosticados nesse hospital foram tumores de ovário $(4,6 \%)$, de abdome e pelve $(4,1 \%)$, de tireoide $(2 \%)$, de partes moles $(2 \%)$ e de intestino $(1 \%)$. Foram realizados também diagnósticos de tumores mais raros na faixa etária pediátrica, como tumor de placenta $(0,5 \%)$ e parótida $(0,5 \%)$ (Tabela 2$)$.

Tabela 2 - Frequência do câncer infanto-juvenil.

\begin{tabular}{cc}
\hline Grupo de diagnóstico & Frequência $\mathbf{( n = 1 9 6 )}$ \\
\hline Leucemias & 43,4 \\
Tumor renal & 16,4 \\
Tumor ósseo & 10,2 \\
Tumor do SNC & 8,2 \\
Linfomas & 7,1 \\
Tumor de ovário & 4,6 \\
Tumor de abdome e pelve & 4,1 \\
Tumor de tireoide & 2 \\
Tumor de partes moles & 2 \\
Tumor intestinal & 1 \\
Tumor de parótida & 0,5 \\
Tumor de placenta & 0,5 \\
\hline
\end{tabular}

Fonte: Araújo MAS, et al., 2020.

Quanto à idade, fez-se a divisão em subgrupos, de acordo com a faixa etária. No primeiro subgrupo, com faixa etária de 0 a 5 anos, $49 \%$ tiveram diagnóstico de leucemia, 32\% de tumor renal, $12 \%$ de tumor de abdome e pelve, $5 \%$ de tumor do SNC e $2 \%$ de tumor de partes moles (Gráfico 1). 
Gráfico 1 - Frequência dos diagnósticos na faixa etária de 0 a 5 anos de idade.

Faixa etária: 0 a 5 anos

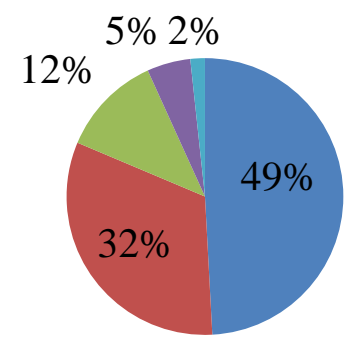

- Leucemias

- Tumor renal

Tumor de abdome e pelve

- Tumor do SNC

- Tumor de partes moles

Fonte: Araújo MAS, et al., 2020.

No segundo subgrupo, com faixa etária entre 6 a 10 anos, 49\% foram diagnosticados com leucemia, 26\% com tumor renal, $19 \%$ com tumor do SNC, $4 \%$ com tumor ósseo e $2 \%$ com tumor de abdome e pelve (Gráfico 2).

Gráfico 2 - Frequência dos diagnósticos na faixa etária de 6 a 10 anos de idade.

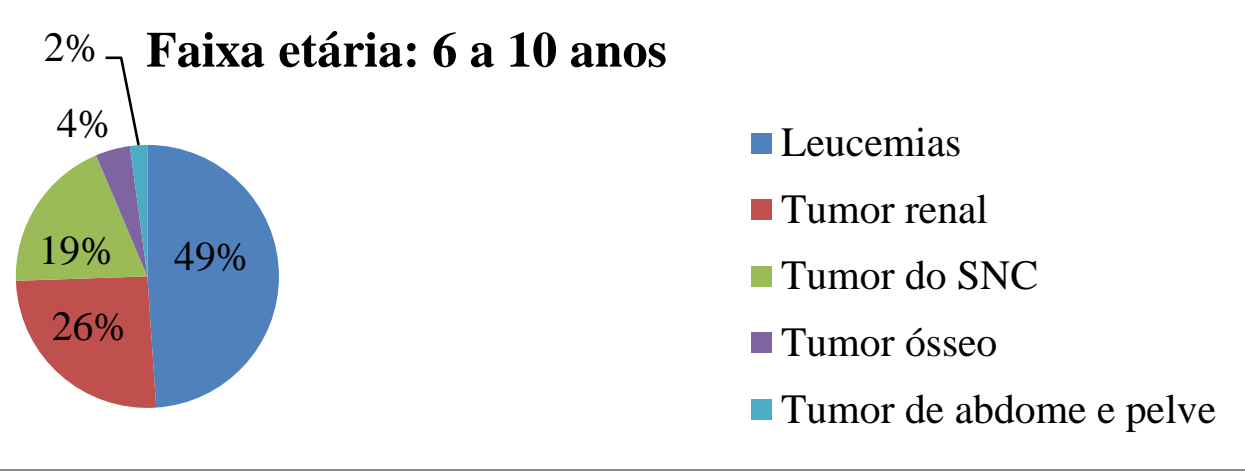

Fonte: Araújo MAS, et al., 2020.

Por fim, no terceiro subgrupo estavam as crianças e adolescentes com idade superior a 10 anos e $37 \%$ foram diagnosticados com leucemia, 20\% com tumor ósseo, 16\% com linfomas, 10\% com tumor de ovário, além de outros diagnósticos mais raros nesse grupo (Gráfico 3).

Gráfico 3 - Frequência dos diagnósticos na faixa etária acima de 10 anos de idade.

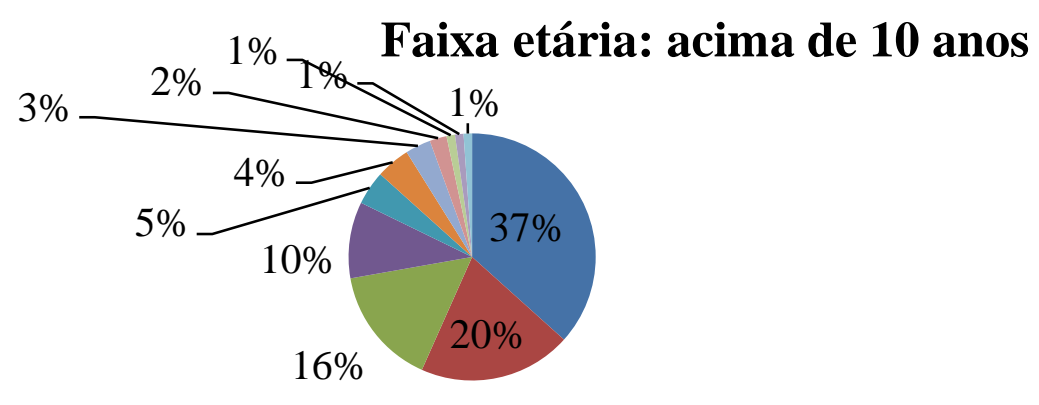

- Leucemias

- Tumor ósseo

- Linfomas

- Tumor de ovário

- Tumor do SNC

Fonte: Araújo MAS, et al., 2020.

\section{DISCUSSÃO}

Este estudo sobre a epidemiologia do câncer infanto-juvenil no estado do Piauí revelou que, no período de janeiro de 2017 a dezembro de 2018, foi feito o diagnóstico de câncer em 196 crianças e adolescentes. Destes, a maioria era do sexo masculino, convergindo com a literatura, que diz que o sexo masculino 
geralmente tem maior incidência de tumores malignos na infância, o que pode demonstrar uma fragilidade constitucional deste sexo, ou até mesmo que haja uma associação de fatores ligados ao cromossomo $X$ (TEIXEIRA AB, et al., 2018).

O estado da Paraíba foi uma exceção, lá foi observado prevalência feminina na maioria dos tipos de cânceres (OLIVEIRA AT, et al., 2019). Estudos realizados nos serviços de oncologia do estado do Rio Grande do Sul e do Piauí também apontaram para predomínio do sexo masculino entre as crianças acometidas (MUTTI CF, et al., 2018).

Ao se descrever os dados relativos a faixa etária, foi verificado que $45 \%$ dos pacientes tinham idade superior a 10 anos, $30 \%$ tinham entre 0 e 5 anos e $25 \%$ entre 6 e 10 anos. No que diz respeito à incidência por faixa etária, a literatura relata que o câncer infantil é mais incidente no primeiro ano de vida, porém essa incidência pode variar nos diversos tipos de câncer, já que alguns são mais prevalentes de acordo com a faixa etária (TEIXEIRA AB, et al., 2018).

No estudo de Mutti CF, et al. (2018), foi observado que a região Norte apresentou maior incidência de câncer infantil na faixa etária até 5 anos de idade e com predominância do sexo masculino. Já no estado do Pará também foi observado maior prevalência no sexo masculino, mas a faixa etária predominante foi entre 8 e 12 anos (HANNA LMO, et al., 2016). Quanto ao quesito procedência dos pacientes, a grande maioria (85\%) dos pacientes eram procedentes do Piauí, de acordo com os dados cadastrados no hospital.

Destes pacientes, $54 \%$ eram oriundos do interior do estado e $34 \%$ de Teresina. Apenas $15 \%$ dos pacientes diagnosticados e tratados no hospital eram de outros estados, mais especificamente do Maranhão. Em estudo realizado em hospital de referência na região norte também foi observado esse padrão de maior quantidade de pacientes oriundos do interior do estado, que precisavam viajar por vezes longas distâncias para ter acesso ao tratamento oncológico (MUTTI CF, et al., 2018). No que diz respeito aos tipos de cânceres mais prevalentes o mais comum foi a leucemia, seguida do tumor renal, tumor ósseo, tumor do SNC e linfomas.

Quando comparamos com um estudo sobre epidemiologia do câncer infantil no estado da Paraíba, que demonstrou a prevalência de leucemias, tumores do SNC e linfomas, respectivamente, nas crianças paraibanas, percebemos que há divergência quanto aos tumores mais comuns em diferentes estados dentro de uma mesma região (OLIVEIRA AT, et al., 2019).

Por outro lado, um estudo realizado no estado do Pará demonstrou que o tipo de neoplasia mais comumente diagnosticada no serviço de referência oncológica do estado foi a leucemia, seguida de nefroblastoma, tumor do SNC e osteossarcoma (HANNA LMO, et al., 2016). Esses dados mostram maior semelhança com os dados encontrados no estado do Piauí.

Além disso, ainda em relação a esse estudo realizado no estado do Pará mostrou-se que houve diagnóstico de cânceres mais raros na infância, como o tumor de ovário (3,45\%), mas as taxas encontradas divergem um pouco das encontradas no Piauí (4,6\%). Conforme dados de um estudo recente que demonstrou a epidemiologia do câncer em crianças e adolescentes no Brasil e no mundo, a leucemia é o tipo de câncer mais incidente, sendo que no Brasil essa taxa varia de $33 \%$ a $26 \%$ dos casos.

Ainda nesse estudo foi revelado que os tumores do SNC ocupam o segundo lugar com $16 \%$ e os linfomas o terceiro lugar com 14\% dos casos (FELICIANO SVM, et al., 2018). Com base nesses dados, percebe-se que o câncer infantil no estado do Piauí difere também das estatísticas nacionais, o que não invalida os dados, já que nesse estudo foram observados apenas pacientes tratados no estado.

A leucemia foi o tipo de câncer infantil mais prevalente no estudo em tela, assim a leucemia do tipo aguda é o tipo de câncer mais frequente nas crianças e se trata de uma neoplasia heterogênea que afeta a medula óssea e que substitui os componentes medulares normais por células imaturas, que podem inclusive se acumular em outros tecidos (OLIVEIRA AT, et al., 2019). Apresenta período de latência curto e os pais geralmente procuram o médico com poucas semanas desde o início dos sintomas, que podem ser palidez cutâneo-mucosa, fadiga, irritabilidade, sangramentos anormais, febre, dentre outros.

Na presença de um ou mais sintomas é necessário realizar investigação inicial com hemograma (BRASIL, 2017). Um estudo realizado no município de São Paulo - SP mostrou taxas de mortalidade da leucemia mais 
altas do que as observadas nos Estados Unidos, com taxa de sobrevida após 60 meses no município para leucemia de $67,63 \%$, sendo maior nos pacientes do sexo feminino e menor naqueles com idade entre 10 a 14 anos (SILVA FF e LATORRE MRDO, 2020).

Dentre os tipos de cânceres mais comum na infância está o tumor renal, sendo o tumor de Wilms a neoplasia renal primária mais comum, com maior prevalência nos meninos. Geralmente o sintoma que leva a criança ao pediatra é o aumento do volume abdominal ou presença de massa abdominal, observados pelos pais. Pode ainda apresentar dor abdominal, hematúria e febre como sintomas (BOUZAS LF e CALAZANS M, 2007).

Os tumores ósseos, também evidenciados como uns dos tipos mais comuns têm como principal representante o osteossarcoma, e se manifestam com dor local associada a aumento de partes moles na região do tumor. Para realizar o diagnóstico precoce é necessário ter atenção aos sinais e sintomas da doença e na suspeita devem ser solicitados exames de imagem.

Frequentemente esse tumor pode enviar metástase para o pulmão levando o paciente a perder peso e desenvolver fadiga e dispneia (BRASIL, 2017). Entre 15\% a 20\% dos pacientes já se apresentam com metástase no momento do diagnóstico, sendo o sítio mais frequente o pulmão (RODRIGUEZ YJ, et al., 2017).

O sarcoma de Ewing é o segundo tumor ósseo primário mais comum entre crianças e adolescentes. Seu principal sintoma é dor com piora progressiva na região do tumor. Pode ter apresentação clínica semelhante à osteomielite (BOUZAS LF e CALAZANS M, 2007). Já em relação aos tumores do SNC os mais comuns são os astrocitomas cerebelares e meduloblastomas.

É indispensável a realização de exames de imagem para investigação. O tratamento é planejado de acordo com o tipo histológico e estadiamento do câncer (NEGREIROS AALV, et al., 2015). No que se refere aos linfomas, também citados como prevalentes nessa faixa etária, estes podem ser classificados em linfomas de Hodgkin e linfomas não Hodgkin e podem acometer qualquer faixa etária.

O linfoma de Hodgkin pode se apresentar com aumento de linfonodo indolor à palpação e de consistência firme, febre, perda de peso, sudorese profusa, astenia e anorexia (SILVA LCM, et al., 2017). O linfoma não Hodgkin é o tipo mais comum e sua apresentação mais frequente é com linfadenopatia cervical dolorosa (GUIMARÃES AC, et al., 2012).

Além disso, um tipo de câncer considerado comum entre crianças, mas sem registro de casos no estado do Piauí no intervalo de tempo estudado, é o retinoblastoma, que se trata do câncer intraocular mais frequente na faixa etária pediátrica.

Possui como apresentação clínica mais frequente a leucocoria e algumas vezes pode se observar estrabismo, dor ocular, diminuição da acuidade visual e proptose (BLANCO WMR, 2013). Para melhor aproveitamento dos dados e identificação do tipo de câncer mais comum por faixa etária, esses pacientes registrados foram divididos ainda em 3 grupos etários: de 0 a 5 anos, de 6 a 10 anos e com idade superior a 10 anos.

No primeiro grupo, o câncer mais diagnosticado foi a leucemia, seguido de tumor renal e tumor de abdome e pelve. No Brasil, nesse mesmo grupo, de acordo com Instituto Nacional de Câncer (INCA), os tumores mais frequentes foram leucemia, neuroblastoma e tumor renal (BRASIL, 2016).

Na faixa etária de 6 a 10 anos, os tumores mais comuns foram leucemia, tumor renal e tumor do SNC, já nas estatísticas brasileiras os mais frequentes foram as leucemias, os linfomas e os tumores do SNC (BRASIL, 2016). Por fim, no subgrupo de crianças e adolescentes com idade superior a 10 anos houve predomínio de leucemia, tumor ósseo e linfomas, e nos dados brasileiros relacionados a esse grupo houve prevalência de leucemia, linfomas e tumores do SNC (BRASIL, 2016).

Portanto, mais uma vez houve divergência dos achados epidemiológicos piauienses quando comparados com o balanço brasileiro, no entanto, como já dito anteriormente, esse fenômeno é decorrente da amostra menor deste estudo e do fato de termos observado apenas uma parcela dos pacientes diagnosticados com câncer infanto-juvenil no Brasil. 
Este estudo teve como limitação o fato de existirem pacientes de outros estados que foram atendidos no hospital de referência para o câncer infantil do município de Teresina-PI, dessa forma tornou-se difícil identificar quais realmente residem no Piauí, visto que muitos pacientes ao adentrarem no setor público de assistência à saúde cadastraram endereços do Piauí, ainda que não sejam de fato residentes no Estado.

\section{CONCLUSÃO}

A investigação da epidemiologia do câncer infanto-juvenil no estado do Piauí revelou que a maioria das crianças e adolescentes diagnosticados com câncer no período avaliado era do sexo masculino, com faixa etária superior a 10 anos, procedentes do Piauí, mais especificamente do interior do estado. A frequência dos diversos tipos de neoplasias diagnosticadas revelou que a leucemia foi a mais comum em todas as faixas etárias. Os outros tipos mais incidentes variaram conforme os grupos etários. Diante disso, o estudo pode contribuir para divulgação de conhecimento sobre a realidade da epidemiologia do câncer infantil no estado do Piauí, com suas peculiaridades, e dessa forma autoridades e serviços de saúde poderão planejar estratégias de triagem e tratamento oportunos para esses pacientes.

\section{AGRADECIMENTOS E FINANCIAMENTO}

Agradecemos à nossa instituição de ensino, Universidade Estadual do Piauí - UESPI, juntamente com o Governo do Estado do Piauí pela oportunidade que nos foi concedida para realização deste estudo e por proporcionar seu financiamento. Após processo seletivo institucional, o estudo foi agraciado com bolsa de iniciação científica da Fundação de Amparo à Pesquisa do Estado do Piauí, que financiou pelo período de um ano a realização da pesquisa durante meados de 2018 a 2019.

\section{REFERÊNCIAS}

1. BENEDETTI GMS, et al. O tratamento do câncer infanto-juvenil: desvelando as vivências dos pais. Revista Latinoamericana de Enfermagem. 2014; 22(3): 425-431.

2. BLANCO WMR. Aspectos clínicos y sobrevida de los pacientes con retinoblastoma atendidos en el Instituto Nacional de Enfermedades Neoplásicas: Enero 2001- agosto 2007. ACTA Med. 2013; 30 (4).

3. BOUZAS LF, CALAZANS M. Tumores sólidos e hematológicos na infância e na adolescência - parte 2. Revista Adolescência \& Saúde. 2007; 4(2): 12-18.

4. BRASIL. Conselho Nacional de Saúde. Resolução no 466 de 12 de dezembro de 2012 . Diretrizes e normas regulamentadoras de pesquisa envolvendo seres humanos. Brasília, 2012.

5. BRASIL. Instituto Nacional de Câncer José Alencar Gomes da Silva (INCA). Monitoramento das ações de controle do câncer em crianças e adolescentes, 2016.

6. BRASIL, MINISTÉRIO DA SAÙDE. Protocolo de diagnóstico precoce do câncer pediátrico. 2017.

7. DELFIN BS, et al. Detecção precoce do câncer infantil em Foz do Iguaçu, PR. Pleiade. 2018; 12 (S1): 29-33.

8. FELICIANO SVM, et al. Incidência e mortalidade por câncer entre crianças e adolescentes: uma revisão narrativa. Revista Brasileira de Cancerologia. 2018; 64(3):389-396.

9. FERNANDES LMS, SOUZA AM. Significados do câncer infantil: a morte se ocupando da vida na infância. Psicologia em estudo. 2019; 24: e39521.

10. GUIMARÃES AC, et al. Linfoma tonsilar em crianças com assimetria tonsilar. Rev. Paul. de Pediatria. 2012; 30(2): 288-291.

11. HANNA LMO, et al. Pediatric cancer: epidemiologic profile of attended children in a reference center in the state of Para. Journal of Research in Dentistry. 2016; 4(3): 100-107.

12. LIMA FFS, et al. Estratégias de intervenção para adesão ao tratamento do câncer infanto-juvenil: relato de caso. Revista Brasileira de Cancerologia. 2018; 64(3): 409-413.

13. LIMA IM. Câncer infantojuvenil: ações de enfermagem na atenção primária à saúde. Rev. APS. $2018 ; 21$ (2): 197 - 205.

14. MARCHI JA, et al. Câncer infanto juvenil: perfil de óbitos. Revista da Rede de Enfermagem do Nordeste. 2013; 14(5): 911-919.

15. MUTTI CF, et al. Perfil clínico-epidemiológico de crianças e adolescentes com câncer em um serviço de oncologia. Revista Brasileira de Cancerologia. 2018, 64 (3), 293-300.

16. NEGREIROS AALV, et al. Aspectos epidemiológicos dos tumores do sistema nervoso central pediátricos em um hospital de referência de João Pessoa (PB) entre 2009 e 2011. Revista Medicina \& Pesquisa. 2015; 1(1): 51-59. 
17. OLIVEIRA AT, et al. Perfil epidemiológico do câncer infantil na Paraíba. Revista Eletrônica Acervo Saúde. 2019; 11(16).

18. RODRIGUEZ YJ, et al. Trombosis em paciente pediátrico con osteosarcoma. Acta Médica Costarricense. 2017, 59(3): 113-116.

19. SANT'ANNA JL, MENDES DMLF. Enfrentamento do Câncer Infantil e Intervenções Psicológicas: Uma Revisão da Literatura. Psic.: Teor. e Pesq. 2019; 35.

20. SILVA LCM, et al. Linfoma de Hodgkin: predominância linfocítica nodular, fisiopatologia e diagnóstico laboratorial. Repositório UniToledo. 2017.

21. SILVA MGP, et al. Tendências da morbimortalidade por câncer infanto-juvenil em um polo de fruticultura irrigada. Cadernos saúde coletiva. 2018; 26 (1): 38-44.

22. SILVA FF, LATORRE MRDO. Sobrevida das leucemias linfoides agudas em crianças no Município de São Paulo, Brasil. Cad. De Saúde Pública. 2020, 36 (3).

23. SPIRONELLO RA, et al. Mortalidade infantil por câncer no Brasil. Saúde e Pesquisa. 2020; 13(1): 115-122.

24. TEIXEIRA AB, et al. Incidência do câncer infanto-juvenil em portadores de Síndrome de Down e avaliação do perfil epidemiológico das crianças internadas no setor de oncologia pediátrica do Hospital Estadual da Criança de Feira de Santana - BA. Anais Semic. 2018; 22.

25. VIEIRA AF, et al. Perfil epidemiológico da leucemia linfoide nas regiões do Brasil. Revista UNILUS Ensino e Pesquisa. 2017; 14(37).

26. WHITAKER MCO, et al. A vida após o câncer infanto-juvenil: experiências dos sobreviventes. Revista Brasileira de Enfermagem. 2013, 66(6): 873-878.

27. WURTTEMBERGER OR. Information and Childhood Cancer. Colombia Médica. 2016, 47(1): 74-75. 